\title{
Migrations et recherches en Allemagne Fédérale
}

In: Revue européenne de migrations internationales. Vol. 2 №2. Novembre. Amériques. pp. 165-192.

Citer ce document / Cite this document :

Hettlage Robert, Khim Francois. Migrations et recherches en Allemagne Fédérale. In: Revue européenne de migrations internationales. Vol. 2 N². Novembre. Amériques. pp. 165-192.

doi : 10.3406/remi.1986.1105

http://www.persee.fr/web/revues/home/prescript/article/remi_0765-0752_1986_num_2_2_1105 
Revue Européenne

des Migrations Internationales

Volume $2-N 2$

Vovembre 1986

CHRONIQUE SCIENTIFIQUE

\title{
Recherches sur les migrations en Allemagne Fédérale
}

\author{
Robert HETTLAGE
}

L.'histoire de la " main-d'œuvre immigrée " en Allemagne, dans ses développements récents, a maintenant une trentaine d'années déjà et c'est pour nous l'occasion de nous demander quels enseignements nous pouvons en tirer. Voilà un propos qui ne déparerait pas un discours officiel, mais il nous incombe ici. d'être plus précis. Tout d'abord. notre intention n'est pas de tirer de cette histoire tel ou tel enseignement, mais plutôt de nous interroger sur les conditions qui président à l'élaboration de vues précises sur un problème donné. Cela nous permettra ensuite d'apprécier la justesse de ces vues et la nature des enseignements qu'il convient éventuellement d'en dégager. Notre étude s'inscrit donc dans la perspective de la sociologie de la connaissance. Elle pose en principe que les structures interactives ont une influence capitale sur la compréhension d'une réalité et que. par conséquent, la réalité des travailleurs immigrés et la politique menée à leur égard sont tributaires de la nature des relations qui s'établissent entre les uns et les autres, du degré de complexité que revêt le processus de compéhension de ce qui est étranger.

Comme l'implique le titre, il sera principalement question, dans la suite de notre développement, de la prise de conscience du problème en République Fédérale d'Allemagne. ce qui n'exclut pas d'autres perspectives, d'autant que les " pays exportateurs " de main-d'œuvre ont dans une certaine mesure réfléchi à ce qu'est la condition d'étranger et ont eu connaissance des phénomènes d'interaction complexes et tendus dans lesquels ils ont engagé leurs ressortissants. 
La catégorisation et la terminologie utilisées pour qualifier les " autres " sont plus éloquentes à elles seules qu'un essai de définition que l'on ne cesserait de corriger ; elles sont même souvent la traduction d'intérêts existants ou, pour le moins, d'impuissance et de maladresse. Le cas des travailleurs immigrés en est une bonne illustration.

\section{LA FORMATION DES CONCEPTS, INDICE DE REFUS DU PROBLEME}

La notion de " politique à l'égard des travailleurs immigrés " est aujourd hui couramment admise dans la recherche internationale qui étudie les migrations, mais nous n'avons pas à en être fiers ; au contraire, car l'emploi de cette formule consacrée traduit fréquemment l'embarras des instances officielles dans leurs rapports avec la communauté, très souvent importante en nombre, des travailleurs migrants résidant dans les pays industrialisés qui les " accueillent " ou les " reçoivent ". Les trente années qui viennent de s'écouler n'ont pu apporter que quelques retouches au tableau, nous allons le voir.

On pourrait dire à peu près la même chose de la recherche sociologique consacrée aux travailleurs immigrés : très souvent à la remorque de l'approche politique de la question, elle ne s'est pas signalée, elle non plus, et pendant longtemps, par l'innovation et le contact direct avec les problèmes qui seuls peuvent faire avancer les choses. En dépit du véritable flot de publications qui existe aujourd hui (et dont on ne peut évidemment rendre compte que d'une manière très sélective), on pourrait classer nombre d'entre elles au chapitre de la stratégie de contournement du problème plutôt qu'à celui de sa compréhension, sans parler de la recherche de solutions.

Le refus, au niveau terminologique, se présente sous deux aspects :

\section{LE REFUS DE CE QUI EST ETRANGER}

Le concept de Gastarbeiter, "travailleur immigré ", caractérise avec une remarquable pertinence les insuffisances des stratégies interactives dans les domaines politique et scientifique, car c'est au fond un mot " piégé " qui dissimule plus qu'il n'éclaire ou plutôt vide de son intérêt une réalité en la réduisant à des aspects partiels qu'il soustrait à la définition au lieu de les y inclure.

La condition de Gastarbeiter suggère que nous avons affaire à un " hôte " (Gast), alors qu'il s'agit en réalité d'un travailleur, situé au plus bas de la hiérarchie statutaire. On considère généralement comme " hôtes " des personnes ou des groupes de personnes que l'on fait bénéficier de stratégies interactives privilégiées, par exemple en veillant particulièrement à leur bien-être et à leur satisfaction - sans que cela soit toutefois dissocié d'un échange d'impressions agréables et de situations où chacun donne de soi une image appropriée à la circonstance. Cela se manifeste par l'attention portée à l'accueil, à la convivialité, par le caractère sacré conféré aux lois de l'hospitalité. Or, dans le cas des travailleurs immigrés, ce sont précisément ces comportements qui sont mis entre parenthèses. On met en avant de toute évidence la deuxième composante du concept, l'état de "travailleur ". La notion de Gastarbeiter subit de ce fait une restriction et une modification de sens 
importantes, car Gast désigne alors des groupes de travailleurs originaires de pays étrangers et d'ethnies différentes dont on peut user avec souplesse au gré des besoins de l'ensemble de l'économie ou de certains de ses secteurs et qu'on peut ensuite refouler, tant il est vrai qu'un hôte ne s'implante pas définitivement mais n'est que de passage, pour le bonheur ou pour le malheur de ceux qui le reçoivent.

Dans ce contexte. on s'attend à ce que l'hôte " poursuive sa route ", sa migration à des fins économiques limitées dans le temps. D'où la stratégie de rotation sur le plan politique. Mais cette vue des choses qui prend pour référence le pays d'accueil peut être à l'origine de formidables tensions quand l'" hôte " n'est pas " celui qui arrive aujourd 'hui pour repartir demain " (Simmel) parce qu'il n'est plus assujetti à l'obligation de rotation, en d'autres termes, à la définition qui lui est imposée du dehors, il oppose une formule d'une autre nature où il définit lui-même sa condition d'"étranger ". I.e concept de Gastarbeiter est inadéquat : pour une autre raison qu'il masque, le phénomène d'altérité aux deux parties concernées. Par leur façon de penser et d'agir, les migrants échappent d'emblée aux repères de la société d'accueil ; celle-ci, les considère comme des étrangers, des êtres curieux, bizarres qui l'incitent à prendre ses distances. Au début, quand on rencontre l'étranger, c'est le plus souvent avec appréhension, voire avec crainte et répulsion. Il faut attendre que se manifestent de part et d'autre les premiers signes de familiarité et les premières marques de confiance pour que puissent fonctionner les normes de l'hospitalité. Or, le fait est que les travailleurs, immigrés sont des étrangers à l'égard desquels on conserve une distance d'orientation ou que l'on fuit. I a référence linguistique à l'hospitalité et au statut d'hôte occulte l'expérience primaire que font les deux parties d'une rencontre culturelle entre "étrangers étranges " (').

Par ailleurs, le concept de migration n'est pas lui non plus assez clair pour permettre à l'analyse de faire d'incontestables progrès, car il évoque un flux d'entrée et de sortie. Du point de vue strictement terminologique, nous nous attendons par conséquent à voir ces étrangers repartir vers d'autres lieux et nous décharger par leur mobilité, d'une partie des problèmes sociaux existants. Mais cela est contredit par les faits. Aujourd'hui les "migrants " sont en majorité depuis plus de dix ans dans notre pays et il est probable quils y resteront pour toute la durée de leur vie active. Sur ce point. on n’a pas non plus attaché suffisamment d'importance à l'établissement de distinctions claires. Divers types de migrations doivent être distingués parce quils recouvrent différents modèles de relations entre celui qui "accueille " et celui qui " est accueilli ". En effet la distinction entre migrants effectuant des rotations de courte durée (cas 1), immigrants définitifs (cas 2) et immigrants de fait encore indécis et vivant dans la perspective illusoire du retour (semi-migration. cas 3), n'est pas sans importance pour la politique et pour la sociologie de la culture, parce que les comportements qu'elle implique correspondent à la définition de situations différentes et à des démarches différentes. Dans les deux premiers cas. la possibilité de retour est envisagée ou plutôt n'est pas exclue ; mais le niveau de conscience est totalement différent chez. les " travailleurs immigrés " (cas 3), qui croient pouvoir se réserver à priori l'échéance à laquelle il faudra décider où et comment vivre sa "vraie vie ". L'étranger dont il est ici question n'est pas celui qui arrive aujourd hui pour repartir demain, mais participe du type auquel songeait Simmel en disant qu' il arrive aujourd 'hui pour rester demain … sorte de migrant en puissance qui, bien quil ne soit pas reparti ailleurs, ne s'est pas encore totalement affranchi de ses velléités migratoires " (Simmel, 1968, p. 685) 


\section{IE REFUS DE IA MACRO ET DE LA MICROHISTOIRE}

Au refus du problème exprimé par la terminologie et à l'obscurité des concepts utilisés dans les milieux politiques et scientifiques ouest-allemands vient s'associer le refus de l'expérience historique et de son arrière-plan, et ce à deux niveaux :

1) Il est fréquent de laisser entendre que la question de l'afflux de main-d'œuvre étrangère ne s'est posée que dans la période d'après-guerre, à l'époque du miracle économique et de la crise qui lui a succédé. Cela revient à exclure de la conscience politique et de celle des chercheurs que, depuis plus de cent ans nous connaissons des migrations collectives, en particulier de travailleurs saisonniers ou permanents originaires de Pologne et d'Italie. Ainsi, sous le Deuxième Reich, le nombre des étrangers vivant dans l'Empire (Reichsausländer) s'était accru de plus d'un million entre 1870 et 1910, passant de 207000 à 1260000 . Et l'on n'a pas tort d'établir un lien entre cette migration et l'essor économique de la Ruhr qui devint alors le premier centre industriel d'Europe. Ces chiffres indiquent non seulement que l'Allemagne était déjà tributaire dans le passé de la main-d'œuvre étrangère. mais aussi que, pour certaines populations, les Polonais par exemple, elle était devenue de fait un pays d'immigration. Cette accumulation d'expérience en matière de processus d'assimilation, d'acculturation et d'intégration n'a pas été exploitée à ce jour (Spies, 1982).

Si l'on fait abstraction du travail obligatoire dans l'agriculture et l'armement durant la Seconde guerre mondiale, l'histoire de l'après-guerre en République Fédérale est liée d'emblée au phénomène d'apport de population : dix millions environ d'expulsés, de réfugiés et de personnes déplacées constituaient globalement $10 \%$ de la population ouest-allemande en 1950. Dans un premier temps, ils purent remplacer la main-d'œuvre étrangère. Ils étaient citoyens allemands, mais les populations résidentes les considéraient au début comme de purs étrangers dont on ne connaissait pas le milieu, dont la langue avait souvent une consonance "étrange " immédiatement repérable, et qui, de ce fait, avaient bien de la peine à se défaire de leurs stigmates d'étrangers. Leur rapide absorption par une économie alors en pleine expansion laissa peu de champ à un débat approfondi sur cette forme de migration concernant des populations qui ne sont pas totalement étrangères. $E_{t}$ surtout, on n'accorda plus l'attention nécessaire au décalage entre intégration et assimilation ; ce qui à tort, comme l'illustre l'embarras que l'on connut par la suite, quand il fallut s'attaquer aux difficultés majeures qu'engendrait la présence des étrangers.

L'activité économique explosa à un rythme tel que le potentiel de main-d'œuvre constitué par la masse des réfugiés ne fut bientôt plus suffisant. Il fallut faire appel à de la main-d'œuvre étrangère, ce qui occasionna un traumatisme dont les conséquences furent naturellement plus lourdes encore. Et comme on n'avait rien retenu du passé économique de l'Allemagne, il fut impossible de puiser à cette source pourtant riche d'enseignements. Immigration et pluriculturalisme furent pour cette raison considérés comme des choses qui n'arrivent qu'aux autres (EtatsUnis !). On crut pouvoir éviter ce genre de problème en décrétant unilatéralement que l'Allemagne n'était pas un pays d'immigration. Mauvais calcul, car le pays d'accueil ne pouvait pas compter sans ses hôtes!

2) La politique et la recherche restèrent coupées de l'histoire pour une dernière raison. Absorbées par les événements déterminants qui marquèrent la 
politique mondiale de notre siècle et par la phase de reconstruction, elles ne se tournèrent pas explicitement vers le vécu et l'expérience migratoire des travailleurs migrants et des immigrants des époques précédentes. On refusa trop facilement de voir que les immigrés ont eux aussi une histoire qu'il leur est difficile d'oublier ou qu'ils ne veulent pas oublier. Comme l'indiquent les histoires de vie, il faut du temps pour que se nouent des identités et des solidarités de groupe. Il faudra également du temps pour qu'elles se dénouent. Par conséquent, on ne peut vraiment pas demander à des étrangers qu'ils opèrent du jour au lendemain un décalage de leurs systèmes de références, car le processus de redéfinition est bien trop complexe, bien trop douloureux et bien trop échelonné dans le temps. Il est en outre peu probable qu'il se déroule selon un axe diamétralement opposé à la situation première. Ce sont là des considérations qui enferment dans d'étroites limites tous les projets d'intégration proposés à la hâte par les hommes politiques.

Par ailleurs, comme l'enseigne l'expérience, nos " concitoyens étrangers " ne sont pas non plus un simple volant de main-d'œuvre disponible à tout moment sans inconvénient majeur pour les uns et les autres. De même que nous ne sommes pas prêts à nous aligner sur les travailleurs immigrés, à cause de notre identité culturelle, de même ne devons nous pas nous attendre de leur part à une volte-face culturelle. Nous oublions trop vite que les objectifs migratoires sont pour l'essentiel de nature économique et qu'un transfert d'identité culturelle n'en est pas la conséquence automatique. Si tant est que cela soit possible - dans la mesure où les résistances de la première génération ne sont pas insurmontables - l'acculturation ne peut se faire qu'au terme de longs processus de rapprochement mutuel. Seules les carences de nos moyens d'analyse peuvent expliquer notre étonnement devant la tendance, en partie délibérée, des populations étrangères à se replier sur ellesmêmes. Les recherches de l'Ecole de Chicago ont du reste enseigné que la " ghettoïsation " est pour ainsi dire une étape " normale " du comportement relationnel, les échanges entre migrants et groupe étranger étant au début nécessairement par l'intermédiaire du groupe, déjà installé, auquel ils appartiennent (Thomas/Znaniecki 1918-21:1 ; Park/Miller 1921, p. 7). Toutefois les conditions dans lesquelles se réalisent ces échanges restent encore à élucider point par point, en tenant compte de la spécificité des populations de migrants (importance numérique, autodéfinition, type de migration, niveau de conscience).

Comme nous le verrons par la suite, la politique et la recherche scientifique se sont d'une certaine manière ralliées aux objectifs avant tout économiques des migrations entreprises par les étrangers et ont à leur instar écarté de leurs préoccupations les problèmes de sociologie culturelle. Or, au fil du temps, nationaux et étrangers se rendirent compte que cette motivation primaire ne pouvait être à elle seule un ferment de cohabitation, voire que " la vie " ne pouvait être tenue à l'écart, ni se limiter à la période des vacances. La " visibilité " des travailleurs migrants, dans la mise et l'apparence se posa en des termes de plus en plus tranchés, la question de leur conception du monde et des possibilités de cohabitation de plusieurs cultures. Comme la recherche allemande n'avait pas bien retenu les leçons du passé, la sociologie était étonnamment mal préparée à définir les principes de ses recherches. Sa propension à considérer le facteur de la main-d'œuvre étrangère dans une perspective réduite, "normale", se constate dans l'histoire récente de la recherche allemande sur les migrations. Il lui fallut du temps et bien des combats 
pour parvenir à une approche plus nuancée; c'est pourquoi, dans certains domaines, elle était en retard de dix à quinze ans sur les réalités.

\section{LES ETAPES DE LA COMPREHENSION ANALYTIQUE ET EMPIRIQUE}

La réflexion et le discours sur un problème donné en fixe les réalités. Les phases successives qui président à la façon de traiter le problème, comportent un certain nombre d'indications qui relèvent de la sociologie de la culture (" cues "): Nelson, 1977) et mettent en lumière la complexité de situations, les difficultés de groupes et les défis politiques qui occupèrent le devant de la scène. Une recherche menée à partir des problèmes économiques et politiques a souvent le souffle court et ne constitue pas vraiment une analyse sociologique. Les trois phases que l'on distingue habituellement (Wilpert, 1984, p. 305 et suiv. ; Gliedner-Simon, 1986) dans la recherche allemande sur les migrations ….. c'est-à-dire, en bref, dans la recherche consacrée aux travailleurs immigrés -- en apporteront la confirmation dans la suite de notre développement :

Au cours de la première phase, celle du miracle économique et de l'accroissement des besoins en main-d'œuvre, la recherche s'incrivit dans une perspective évolutionniste résolument optimiste. Marquée par l'" economic welcome ", elle était mêlée d'admiration inavouée pour ces étrangers et pour la facilité avec laquelle s'équilibraient les intérêts économiques des parties engagées. La migration de travail était considérée comme la chance offerte à l'économie nationale et aux pays exportateurs de main-d'œuvre, pour la première de combler une lacune, pour les seconds de réduire un excédent et d'équilibrer leurs budgets par les transferts.

Au cours de la seconde phase, lorsque la conjoncture amorça lentement un palier, on commença peu à peu à prendre conscience des charges que faisait peser sur l'économie et la société l'afflux massif de populations étrangères. I.'approche scientifique du problème sinfléchit en conséquence.

Enfin, lors de la troisième phase, le phénomène primordial a moins été le nombre important d'entrées que le séjour permanent. La migration est devenue une immigration de fait. La République Fédérale d'Allemagne est-elle vraiment un pays d'immigration? De nouveaux groupes ethniques se sont-ils fixés, constituant anarchiquement des "réalités " désormais incontournables pour le pays d'accueil? Ainsi, c'est seulement vers la fin des années 70 que, dans toute leur acuité, se sont posées à la sociologie les questions de lidentité culturelle des nationaux et de la population immigrée, de la signification que pourrait revêtir à l'avenir la politique à l'égard des étrangers, de l'opportunité et de la façon de contrecarrer l'évolution vers le pluriculturalisme et du mode d'intégration souhaitable.

Tel est le paysage dans lequel s'inscrit la lente modification de la recherche. Un aperçu de la littérature scientifique consacrée au sujet viendra le préciser dans la suite de notre exposé, lequel, sans prétendre à l'exhaustivité, fera porter l'accent sur les traits caractéristiques des travaux de recherche. 


\section{LA PHASE ECONOMIQUE (1955-1972): LETRANGER FACTEUR DE FLEXIBILITE DANS LA PRODUCTION}

\section{L'euphorie du recrutement}

Pour l'analyse des problèmes migratoires dans l'histoire de l'Allemagne d'après-guerre, l'année-clé est 1955 , date à laquelle fut conclu avec l'Italie le premier accord sur l'envoi de travailleurs immigrés. Mais c'est seulement dans les années 60 que cette période acquit son véritable dynamisme, lorsque furent conclus avec d'autres pays du Sud de l'Europe ou avec des pays non-européens des accords analogues sur le recrutement de main-d'œuvre et que des bureaux de recrutement furent mis en place dans ces Etats.

L'intention qui présida à tous ces accords passés avec l'Espagne et la Grèce (1960), la Turquie (1961), le Maroc (1963), le Portugal (1964), la Tunisie (1965) et la Yougoslavie (1968) était de faire venir, pour une durée limitée, une main-d'œuvre destinée à soutenir l'économie allemande et celle du pays étranger (Reimann/Reimann 1979, p. 66). Dans l'esprit de leurs initiateurs cette main-d'œuvre n'était qu'un facteur de production garantissant liberté de manœuvre et souplesse d'intervention. C'est pourquoi le recrutement était régi par le principe de rotation. Pour pouvoir renvoyer cette main-d'œuvre chez elle avec la même souplesse, elle ne devait à aucun moment être en mesure de remplir les conditions requises pour le séjour permanent et la naturalisation.

Le libellé des accords présentait des différences, dans la mesure où les ressortissants de la C.E.E. bénéficiaient, aux termes des Traités de Rome (1958), de la liberté d'installation et où il n'était plus nécessaire de leur délivrer de permis de séjour. Quantité de contrats de travail furent établis sur le modèle italien. L'autorisation de séjour d'au moins 5 ans fut renouvellée tant que l'intéressé pouvait justifier d'un emploi. Actuellement, le migrant même au chômage ne peut être refoulé, à moins qu'il ne soit en infraction avec la réglementation ou constitue une menace pour l'ordre public. En revanche, le statut juridique des ressortissants hors C.E.E. est plus précaire. Ils tombent sous le coup de la loi sur les étrangers qui stipule qu'une autorisation de séjour ne peut être accordée que si elle est compatible avec les intérêts de la République Fédérale, l'application des textes étant laissée à l'appréciation de l'administration. En outre, l'autorisation de séjour peut être restreinte à certaines régions ou à certaines entreprises. Sa durée est normalement limitée à 2 ans, mais peut être portée à 3,4 ou 5 ans. Après un séjour de plus d'un an en République Fédérale, le migrant peut faire venir sa famille. Cette disposition, étendue ultérieurement à la Turquie, mit davantage en évidence le " problème des étrangers " dans les années qui suivirent et engendra, sur le plan social, des difficultés et des séquelles qu'il ne fut plus guère possible d'évacuer. (Nous n'aborderons pas ici les réglementations spéciales adoptées ultérieurement).

\section{La perception initiale du problème par les migrations}

Partant des besoins en main-d'œuvre de l'économie, la recherche allemande de l'époque privilégia l'étude des coûts et des avantages que représentaient les migrations internationales (Lohrmann, 1974 ; Weidacher/Lopez-Blasco, 1982), considérant soit les effets positifs d'un volant de main-d'œuvre flexible, soit les incidences 
de l'accroissement de la concurrence sur la productivité du travail (Kisker, 1979). Les syndicats adoptèrent eux aussi cette perspective.

La même référence au marché du travail se retrouva dans les études, vivement encouragées par les Länder, sur les structures de l'emploi locales. Un bon exemple en est l'étude de Mehrländer (1969), où l'explication de l'impulsion donnée à la croissance par la migration est que le recours aux travailleurs immigrés permet d'éviter les frais de formation professionnelle et les charges (assurances sociales) - argument qui allait perdre toute force avec le regroupement des familles. Les études de l'Office Fédéral du Travail (Bundesanstalt für Arbeit, BfA) sur les conditions d'existence et les conditions de travail des travailleurs étrangers $(1970 ; 1973)$ doivent être considérées, elles aussi, comme un ensemble de documents relevant plutôt de la politique de l'emploi que de l'analyse sociologique.

Seules quelques études portant sur des villes particulièrement concernées comme Francfort et plus tard Berlin infléchirent leur perspective vers la sociologie (Borris, 1973) et s'interrogèrent sur les conditions d'existence des étrangers, sur les impératifs qu'implique une infrastructure adéquate et - prenant pour base le statut juridique des étrangers - sur le potentiel d'intégration de la ville (Peters, 1982).

Les recherches sociologiques consacrées aux comportements et aux capacités d'adaptation des travailleurs immigrés furent rares à l'époque. Seules trois études, que l'on peut considérer comme des exceptions, méritent ici d'être soulignées : celles de U. Kurz, de G. Albrecht et de H.J. Hoffmann-Nowotny.

L'étude de U. Kurz (1965) sur les baraquements des immigrés italiens à Munich est la toute première à manifester une conscience du problème qui participe de la sociologie culturelle. Elle met en évidence que la perspective du retour, l'isolement forcé des migrants et la proximité géographique du pays d'origine ont permis de maintenir dans une large mesure le système de valeurs traditionnel, au point qu'on ne peut s'attendre, dans le meilleur des cas, qu'à une "adaptation partielle " de ces étrangers à la culture du pays d'accueil.

La sociologie de la mobilité géographique de G. Albrecht (1972) s'interroge sur le choix du moment où s'engage un mouvement migratoire et voit en ce dernier un ensemble de moyens fonctionnels destiné à désamorcer, dans le pays d'origine, la recrudescence de crises et de luttes pour des ressources limitées. Cela dit, l'auteur ajoute que les migrations sont aussi un élément du changement social, mais il ne se demande pas si - et dans quelle mesure - elles peuvent être également un élément de stabilisation sociale.

Les études de H.J. Hoffmann-Nowotny $(1970,1973)$ furent à l'époque les plus exigeantes sur le plan théorique et connurent le plus grand rayonnement. S'appuyant sur des données collectées en Suisse, son argumentation théorique se donne pour objectif de prouver que les inégalités de pouvoir et de prestige résultent de tensions structurelles. Les migrations apparaissent comme un moyen possible de résorber ces tensions. Mais elles nécessitent une adaptation à des normes et, dans ce cas, la préférence revient à l'intégration (dispersion institutionnelle) au détriment de l'acculturation (dépassement des différences culturelles). Les travailleurs immigrés sont alors obligés de conformer leurs aspirations aux possibilités qu'ils ont de les 
réaliser, c'est-à-dire, soit abandonner leurs espoirs et chercher une consolation dans un éventuel retour, soit ramener leurs aspirations au niveau qu'ils occupent dans la société et compenser les tensions par la consommation - le résultat final étant le "déclassement néoféodal ".

\section{LA PHASE SOCIO-POLITIQUE ET SOCIO-PEDAGOGIQUE (1973-1979) LES ETRANGERS PESANTEUR SOCIO-ECONOMIQUE}

\section{Les conséquences de l'arrêt du recrutement}

A la fin de l'année 1973, la phase de relative insouciance se termina brutalement, bien qu'une première série de modifications profondes fût intervenue dès 1969 dans la situation sociale des travailleurs immigrés et dans la manière d’aborder le problème :

Economiquement, le principe de rotation s'était avéré trop coûteux et donc inefficace (Dohse, 1981). Il n'était plus possible de tirer parti des efforts qu'il avait été indispensable de faire en faveur de la formation des travailleurs immigrés (training on the job) si ceux-ci devaient repartir. Les opportunités de formation furent donc l'une des causes de l'allongement important du séjour des étrangers. Ne disposant pas de solution de rechange sur le plan économique, la République Fédérale devint pour nombre d'étrangers qui y étaient venus, sinon un pays d'immigration, du moins un " pays de séjour permanent " (Reimann, 1984, p. 66). Sur les 4500000 " hôtes" étrangers que compte environ la RFA à l'heure actuelle, plus de la moitié est arrivée il y a plus de dix ans. Hésitant entre l'idée de rotation et d'intégration, l'étranger n'avait le choix qu'entre une durée de séjour limitée associée aux aléas du retour dans son pays (débouchés professionnels, dépaysement partiel) et l'installation à demeure en terre étrangère en dépit de la précarité de sa situation et de ses multiples difficultés d'insertion (langue, reconnaissance sociale et statutaire, accès à l'information, marginalisation, etc.).

Mais l'événement déterminant de cette phase fut la rupture conjoncturelle enregistrée au début des années 70 à la suite de ce qu'il est convenu d'appeler le choc pétrolier. Elle entraîna un brusque revirement de l'opinion publique. Dès lors, les esprits furent hantés par l'idée que la migration n'avait pas que des avantages et que, dans certaines conditions, elle constituait même une pesanteur énorme pour l'Etat et l'économie. Les syndicats commencèrent eux aussi à s'émouvoir, car ils craignaient que la crise ait de fâcheuses répercussions sur la sécurité de l'emploi de leur clientèle attitrée. Rien qu'en 1973, on avait embauché plus de 300000 personnes supplémentaires. Le gouvernement fédéral était mis au pied du mur et, le 22 novembre 1973, il chargea l'Office Fédéral du Travail de suspendre immédiatement le recrutement en Turquie, en Yougoslavie, en Espagne, au Portugal, en Grèce, en Tunisie et au Maroc et de fermer ses bureaux dans ces pays.

Depuis cette époque, l'opinion n'a jamais cessé de se demander si cela avait effectivement permis de résoudre les problèmes du marché du travail en RFA ou pouvait un jour les résoudre. Une fraction importante de la population pensa quil fallait renvoyer chez eux les travailleurs originaires de pays tiers. Les partenaires sociaux préférèrent jouer la carte de la fluctuation volontaire associée à l'interdiction de retour en RFA pour ramener le nombre global des travailleurs étrangers à un niveau acceptable sans avoir à prendre des mesures administratives de coerci- 
tion. Mais la conséquence immédiate de l'arrêt du recrutement fut l'augmentation des regroupements familiaux. Tout au moins, la tendance déjà amorcée avant 1973 ne fit que s'intensifier, car il était pratiquement exclu de retrouver un emploi une fois après avoir quitté " définitivement " la République Fédérale. On fit donc le choix de rester dans le pays d'accueil et de s'y installer en dépit des incertitudes de l'emploi.

L’afflux massif d'immigrés, l'augmentation de la durée de leur séjour et le regroupement des familles focalisèrent finalement encore plus l'attention sur la population étrangère résidente. En 1974, les Allemands jusqu'alors persuadés de " rester maîtres chez eux " furent ébranlés dans leur conviction lorsqu'il s'avéra que $18 \%$ des naissances d'une même année étaient issus de la population étrangère. Dans certaines villes, le pourcentage atteignait même 35 à $45 \%$ (!) (Munscher, 1979), d'où une série de mesures visant à limiter l'immigration dans certaines régions.

A la charge supportée par le marché du travail venait s'ajouter un autre problème : il fallait réserver chaque année 40000 à 60000 postes de travail et de formation pour les jeunes étrangers en âge de travailler, alors quill était déjà nécessaire de créer un million d'emplois supplémentaires pour les jeunes nationaux issus des années de forte natalité. Pour la première fois, on prit pleinement conscience des tâches qu'imposait à la société l'émergence d'une seconde génération d'étrangers. On craignait à juste titre la montée de tensions explosives de part et d'autre si l'on ne parvenait pas à trouver de nouvelles solutions - problème dont les données n'ont rien perdu de leur actualité dans la troisième phase (Lebon/Falchi, 1980, p. 548 et suiv.; Gökalp, 1984, p. 487 ; Schierup, 1985, p. 21 et suiv.).

\section{La différenciation des objectifs principaux}

Les modifications successives de l'attitude vis-à-vis de la migration de la population active ainsi que la sensibilisation globalement plus forte à la présence étrangère ont donné lieu à une série de travaux qui tranchent nettement sur les précédents par leur orientation thématique. Ils se répartissent en deux grands groupes : ceux consacrés à l'infrastructure sociale (conditions d'habitation, choix proposés en matière d'éducation) et les travaux consacrés à la situation particulière de la seconde génération (formation professionnelle, délinquance, pédagogie à l'usage des étrangers).

\section{L'INFRASTRUCTURE SOCIALE ET LE PROBLEME DE LA SEGREGATION}

A la fin des années 60, l'étude d'infrastructres adaptées aux besoins des étrangers se concentra principalement sur la question du logement. Les anciennes cités composées de baraquements pour travailleurs immigrés avaient certes disparu, mais les premières enquêtes dressèrent un tableau qui restait préoccupant (Schildmeier, 1975 ; Zieris, 1977). Outre les difficultés résultant de la recherche d'un logement, du prix élevé des loyers et de la qualité inférieure des locaux habités par les étrangers, ce qui attira surtout l'attention des chercheurs fut la forte concentration de travailleurs immigrés dans certains quartiers, certaines rues et certains immeubles. Ceci était particulièrement remarquable dans le quartier berlinois de 
Kreuzberg où se vérifiait, d'une manière saisissante qui frappait l'opinion. le cycle "infiltration, invasion, succession" ("white flight") bien connu par les études faites aux Etats-Unis (Hoffmeyer-Zlotnik, 1977 ; pour la ville de Vienne : Leitner, 1983). Deux questions retinrent principalement l'intérêt.

I a première était de savoir si les conditions d'habitation sont un indicateur du degré d'intégration. Selon Ipsen (1978), il n'est pas possible d'établir un lien direct entre durée de séjour et intégration matérielle, parce que les conditions de logement de ces populations restent souvent inchangées pendant des années. On serait plutôt tenté de conclure qu'avec le temps, leurs exigences diminuent, le mirage du retour contribuant d'une manière non négligeable au dépassement des tensions. Il semble que l'apathie soit un refuge nécessaire pour vider de son contenu explosif la discrimination ethnique qui règne sur le marché du logement.

La deuxième question visait à établir si la discrimination est voulue ou imposće. Sur ce point, Diricks Kudat (1975) mirent en lumière, à partir d'un échantillon d'ouvriers turcs et yougoslaves établis à Berlin, que ce sont les conditions d'environnement et non les préférences personnelles des migrants qui ont le plus de poids. Ces conditions regroupent, en plus de considérations matérielles, les comportements discriminatoires de la population autochtone (Wurtinger/Baatz, 1980) et ce sont elles qui déclenchent finalement -... troisième facteur - - un processus au cours duquel les migrants se dotent d'organisations socio-culturelles autonomes dans des enclaves ethniques. Heckmann fait cependant remarquer que, dans un modèle migratoire comprenant plusieurs phases, la ségrégation pourrait n'être qu'une première étape transitoire (Neckmann, 1980, p. 105 et suiv. : Heckmann, 1981). Le comportement ségrégatif a de son côté une incidence négative sur le secteur sociopédagogique. En effet, comme le cycle de succession des travailleurs immigrés s'est concentré essentiellement sur des zones déjà peu structurées qui furent ensuite abandonnés par la population autochtone, le sous-équipement de ces quartiers en matière éducative est resté ce qu'il était (Haas Grugelke et coauteurs, 1980).

\section{I.A SECONDE GENERATION}

Désormais, le séjour permanent des migrants imposait aux pouvoirs publics une tâche qui ne pouvait se limiter à présenter à la première génération de travailleurs et de résidents, qui s'était développée à l'étranger, des propositions d'intégration convenables. Il devenait par la force des choses encore plus important -... et apparemment aussi plus prometteur ... de concentrer ses efforts sur la seconde génération née, ou du moins venue en bas âge en RFA. Etant donné que ces jeunes étrangers, à la différence des immigrants n'acquièrent pas, comme par exemple, la citoyenneté par la naissance aux Etats-Unis, mais qu'inversement ils se sentent plus proches du pays d'accueil que la génération de leurs parents, leur existence reflète un déchirement que notent toutes les recherches consacrées au sujet, même quand leurs conclusions sont ambiguës. L'analyse se pencha principalement sur cinq aspects de la question.

\section{Cursus scolaire et perspectives professionnelles}

Il apparaît d'une part, que les écoles et les enseignants allemands n'étaient pas du tout préparés à l'afflux massif d'écoliers étrangers (Boos-Nünning Hohmann - 
Reich, 1976). D'autre part, en raison de leur socialisation "éclatée ", les jeunes étrangers eux-mêmes ne sont pas souvent non plus en mesure de profiter pleinement des possibilités offertes par l'école, soit à cause de leur double handicap linguistique, soit à cause de leur isolement dans les classes des écoles allemandes ou encore du mécontentement de leurs parents à propos de certains enseignements considérés par eux comme inadaptés (cf. à ce sujet la situation des jeunes filles turques : Weische-Alexa, 1978 ; Akpinar, 1979 ; Rosen/Stuve, 1985). Tout cela entraîne des anomalies notables du comportement et explique pourquoi le nombre d'enfants qui interrompent leur scolarité est si élevé (Birkenfeld, 1982 ; Deutsches Jugendinstitut, Institut Allemand de la Jeunesse, 1980 ; McRae, 1982) (2).

Outre les difficultés scolaires, la qualification des enfants d'origine étrangère dans la compétition qui les oppose aux jeunes allemands (Bundesjugendkuratorium, Conseil Fédéral de la Jeunesse, 1976) retint l'attention. Comme le taux de chômage était comparativement beaucoup plus élevé chez les jeunes étrangers que chez les jeunes nationaux, le gouvernement fédéral se préoccupa de cette catégorie en difficulté en instituant un " programme jeunes " qui lui fut spécialement destiné.

C'est ainsi qu'à l'analyse des carences de la formation professionnelle, de la pénurie des postes d'apprentissage et de la concurrence où les jeunes étrangers risquent d'avoir le dessous, vint s'ajouter l'évaluation des programmes de recherche. Ces programmes furent complétés par l'étude des attentes des jeunes étrangers. Les jeunes étrangers imputent fréquemment leurs handicaps à des défaillances personnelles et que le niveau des aspirations de la seconde génération, indépendamment du comportement relationnel et de la durée du séjour, est généralement bien plus élevé qu'on ne l'imagine (Wilpert, 1980). Les différences de culture semblent avoir moins de poids en la matière, car les cultures étrangères qui nous intéressent ont une éthique de l'efficacité qui n'est manifestement pas sans rappeler celle des sociétés industrielles occidentales (Schrader/Nikles, 1979², p. 98 et suiv.). Ce sont plutôt l'inadaptation du système familial et le manque d'information qui seraient à l'origine des écarts constatés (Papaioannou, 1983 ; Sassen, 1984).

\section{Structure familiale et constitution d'une identité "éclatée "}

Concentrer l'attention sur les problèmes scolaires comme on le fit au début ne suffit pas à appréhender convenablement les difficultés et les conflits de la seconde génération. De plus en plus, les problèmes concernant la socialisation des enfants d'origine étrangère au sein de leurs familles passèrent au premier plan.

Malgré l'actuelle rareté des études consacrées aux familles de migrants (Renner, 1975 ; Schrader/Nikles, 1979², Mertens/Akpinar, 1977 ; Wilpert, 1980 ; Ligouras, 1981), on voit cependant se dessiner une transformation des structures familiales et une redistribution des tâches. Ainsi la conception traditionnelle du rôle des parents et des enfants opère une mutation au profit des enfants socialement plus compétents, tandis que, d'autre part, des différenciations s'ébauchent dans les rôles respectifs de l'homme et de la femme. Kudat (1975) a pu montrer à Berlin que les normes patriarcales dominantes et le contrôle social tendent à s'affaiblir et que cette évolution va de pair avec l'acquisition autonome d'un revenu par la femme et les enfants. Cela s'accompagne de modifications qui touchent la façon de concevoir la division du travail, le statut de la femme au sein de la famille et les aspirations 
personnelles (Schiffauer, 1984 : I.ey, 1979). Mais il faut dire que nous manquons encore à l'heure actuelle d'études approfondies sur ces sujets.

En prenant pour point de départ les conflits de cultures qui se produisent vraisemblablement dans les familles, on distinguera grossièrement deux types de structures familiales: un type rigide qui se conforme dautant plus aux normes traditionnelles (ce quil est convenu dappeler "returquisation ") quil se trouve transplanté en milieu étranger hors de son univers, et un type plus souple qui se prépare plus sciemment et plus franchement à vivre en permanence à l'étranger (Neumann, 1980). Mais les stratégies quils supposent l'un et l'autre sont encore largement inexplorées.

Ces questions débouchent directement sur l'analyse de l'identité culturelle que se construisent les résidents permanents de la première et de la seconde génération dans des conditions de socialisation biculturelle. I. 'hỵpothèse retenue à l'origine fut qu'un décalage dans la façon de se représenter des valeurs entraîne obligatoirement des conflits de comportement (Boos- Vünning Hohmann. 1976) et, au niveau de la seconde génération, une distanciation par rapport aux rôles traditionnels (Twenhöfel, 1984). D’autres études avancent que la faculté d'adaptation et l'identification avec le pays d'accueil sont fondamentalement déterminées par le lieu d'enracinement culturel de la petite enfance (Schrader Nikles, 1979, p. 67 et suiv.). I. 'assimilation comme stade ultime d'identification avec certaines valeurs ne serait done accessible qu’à ceux qui sont nés en RFA (" nouveaux Allemands").

\section{Comportements déviants}

Tout cela permit bien souvent de conclure que les conflits de valeurs, d'éducations et de comportements expliquent pour l'essentiel le niveau apparemment plus élevé du taux de criminalité chez les enfants et les jeunes d'origine étrangère. Une analyse plus précise indiqua cependant que cette conclusion. si tant est qu'on puisse la retenir, ne s’applique quà certains délits (délits sexuels, délits de violence) et à certaines classes d’âge (de 14 à 18 ans et de 18 à 21 ans). Dans l'ensemble. la délinquance est même légèrement plus élevée chez les Allemands que chè les étrangers (Albrecht Pfeiffer. 1979, p. 32).

La recherche des causes de la criminalité (Bingemer et coauteurs, 1970); Bielefeld et coauteurs, 1982) fait apparaître que la seconde génération est à bien des égards une catégorie en difficulté. I a discrimination scolaire et professionnelle se double chez elle en effet de conflits culturels intrafamiliaux. Son isolement à l'extérieur est donc renforcé par l'incapacité dans laquelle se trouve la famille d'assister l'adolescent dans ses difficultés d'orientation. I es frustrations et les contraintes trop lourdes qui pèsent sur le psychisme peuvent facilement dégénérer en agressivité, d'autant plus que la situation tendue du marché du travail et lincertitude des perspectives d’avenir recèlent un potentiel supplémentaire de conflits.

Pour tout ce qui concerne les problèmes de déviances, on ne peut cependant pas écarter l'éventualité d'une "illusion d'optique, car, comme dans le cas d'enfants de milieux sociaux défavorisés, la catégorisation opérée ici par l'autorité judiciaire n'est vraisemblablement pas sans poser de problèmes. Ces incertitudes, qui pèsent lourdement sur les données dont nous disposons, n'ont pas encore été levées. 


\section{LA PHASE DE POLITIQUE D'INTEGRATION (A PARTIR DE 1979) : I.ES ETRANGERS ET LA DIFFERENCIATION CULTURELLE AUSEIN DE LA SOCIETE}

\section{I.e séjour permanent, une voie menant vers l'intégration ?}

Phénomène de masse désormais accompagné de fortes tensions culturelles et de conflits de comportements entre la première et la seconde génération de travailleurs immigrés, l'établissement des étrangers remit en cause, une fois de plus, la perspective adoptée jusqu'alors sur le plan politique pour appréhender le problème. Bien que la République Fédérale réitérât son refus d'être considérée comme un pays d'immigration, les esprits acquirent peu à peu la conviction qu'il était indispensable de promulguer à moyen terme une politique globale en faveur des étrangers. Sous la forme d'une minorité relativement stable, la population étrangère active et résidente était sur le point de s'installer de facto en Allemagne. Il ne suffisait plus aux pouvoirs publics de prendre des mesures destinées à corriger, à court terme, les infrastructures sociales, du simple fait que les pays d'origine comme la Turquie refusaient totalement qu'une politique de rotation, en réexportant chez. eux les problèmes du marché ouest-allemand du travail, les privât de leurs atouts. C'est ainsi que la Turquie avait obtenu assez. tôt le bénéfice d'un accord de libre circulation analogue à celui de la CEE sur lequel il n'était plus question pour elle de revenir mais dont l'application (1986) avait dû être provisoirement reportée en raison des énormes avantages économiques préalablement consentis par la République Fédérale. Cette nouvelle conscience de soi est un trait symptômatique que l'on retrouve au sein des groupes de travailleurs immigrés. Ceux-ci sont de moins en moins disposés à s'entendre dire qu'ils ne sont qu'un volant de main-d'œuvre et qu'ils constituent un "problème social ". Même s’ils ont fondamentalement le désir de retourner dans leurs pays d'origine, les migrants et leurs familles ne veulent pas se voir imposer du dehors la date de leur retour ou le cadre dans lequel doit se dérouler leur existence bi-culturelle. La longueur du séjour a aussi introduit un changement dans les consciences. Leur objectif prioritaire n'est désormais plus uniquement de vaincre la précarité de leur situation économique ; ce qui importe à leurs yeux, c'est aussi la modification des conditions de formation, la libre circulation, l'acquisition d'un statut social et l'égalité au regard des institutions. En un mot, ils veulent aussi obtenir le " pouvoir de définir " ce qui leur paraît être les chances de leur double appartenance et ce qui leur semble néfaste dans leurs contacts avec la culture dominante de la société d'accueil. Et là encore, il ne s'agit pas seulement de solliciter une fois de plus le concours des pouvoirs publics; il y va tout autant de la reconnaissance de leur droit à user de leurs libertés nouvelles pour marquer une distance par rapport au pays d'accueil sans obligatoirement se faire taxer d'ingratitude. Ils veulent en quelque sorte faire respecter leur originalité culturelle sans avoir pour autant à décider si elle doit le cas échéant s'accompagner de l'abandon du droit de séjour prolongé en Allemagne.

Ces changements dans les mentalités et les comportements des migrants placent le pays d'accueil devant un ensemble de questions jusqu'alors complaisamment éludées, à savoir : quelle forme d'intégration est-on en mesure ou en droit d'exiger des travailleurs immigrés ? Quelle marge d'autonomie culturelle et de distanciation par rapport aux normes peut-on leur accorder? Ou, en d'autres termes : que signifient aujourd'hui intégration et politique d'intégration? 
C'est précisément le point sur lequel se heurtent de plein front des intérêts et des représentations culturelles divergents, tant chez. les autochtones qu'entre autochtones et étrangers.

Quand on aura admis que l'intégration concerne la participation aux institutions, l'insertion sociale, l'accès à égalité de droits à des réalités convoitées (pouvoir. prestige). la protection des individus, l'instauration de rapports de sympathie, il restera encore à savoir par quelle voie on y parviendra : voie unique ou pluraliste ? Dans le premier cas, l'intégration aura pour terme l'assimilation et l'absorption culturelles, dans le deuxième on n'exigera pas cette conformité aux normes, réservant ainsi l'avenir et la question de la réémigration. Dans les deux hypothèses, se pose le problème de savoir s'il ne sera pas nécessaire d'admettre une intégration ethnique "endogène " (insularisation). En effet, les formes de sous-culture sont un facteur d'intégration. dans la mesure où elles potentialisent les énergies et les moyens du groupe (par exemple par la représentation de ses intérêts, par une politique d'information) et permettent d'éviter le verrouillage culturel, les comportements de repli, la révolte et l'agressivité (Schult7, 1979, p. 9 ; Elwert, p. 717 et suiv.).

Faut-il accepter, voire encourager cette évolution? Sur le plan politique, c'est une décision qui, pour le pays d'accueil, ne sera évidemment pas facile à prendre.

\section{Les acquis récents de la sociologie des migrations et leur apport dans la perception du problème}

Une fois encore, les investigations de la sociologie s'infléchirent en fonction des modifications de la situation politique. D'une part, la question se posa de savoir si et dans quelle mesure des ethnies nouvelles s'étaient constituées sur le territoire national à partir de populations immigrées. D'autre part, il fallut plus largement tenir compte des réactions hostiles qui se manifestaient che $\%$ les autochtones sous la forme d'attitudes xénophobes. Ajoutons que cela remit à l'ordre du jour le débat sur la réémigration.

\section{I'ASSIMILATION DES MINORITES ETHNIQUES}

Il est généralement admis en Allemagne qu'avec la "sociologie des migrations " de H. Esser (1981) une étape importante a été franchie dans l'approche sociologique du problème de la migration. Pour $\mathrm{H}$. Esser, contrairement à Hoffmann-Nowotny, le processus déterminant est l'assimilation. Par assimilation, il entend le degré de similitude entre migrants et population autochtone au niveau cognitif, identitaire, mais aussi social (relations interethniques) et structurel (conformité des statuts). Son explication qui débouche sur une théorie de l'action admet au départ que les migrants ne peuvent vaincre le manque d'assurance de leur comportement que s'ils sont détenteurs de connaissances et d'aptitudes sans lesquelles il est impossible de mener une vie satisfaisante dans la société d'accueil. Ils doivent donc s'assujettir à un processus d'acquisition qui embrasse les domaines les plus divers de la culture d'accueil (langue. rôle professionnel, représentation des valeurs, interprétation des normes. etc.). I.e mécanisme de cette resocialisation sont déterminés par des facteurs personnels (niveau de motivation. connaissances) et des 
facteurs écologiques (ouverture du principe au monde extérieur, absence d'interdits, valorisation du comportement d'assimilation). Tous les processus d'acquisition se dérouleraient selon le théorème suivant : l'acteur choisit le type d'action qui. aux yeux de son environnement, lui donne le plus de chances d'atteindre son objectif, compte tenu de ses motifs, de ses capacités et des représentations qu'il se fait des opportunités, des obstacles et des alternatives qui se présentent (Hill, 1984. p. 89).

Une étude consacrée, sur ces bases, au comportement des cinq populations immigrées majoritaires en RFA (Kremer'Spangenberg, 1981) a montré que les hésitations du comportement, les sentiments de discrimination et le freinage des aspirations sont étroitement liés aux résistances à l'assimilation.

Inversement, une enquête de Koch Arzberger (1985) indique que l'intégration des étrangers n'est pas très avancée dans les domaines du travail, de l'habitat et de la politique, mais que les réseaux qui se tissent dans les secteurs de l'amitié personnelle, du mariage et de la vie associative permettent de brosser un tableau plus réjouissant (p. 176 et suiv.). I.élaboration par Hill (1984, p. 159 et suiv.) d'un classement des comportements relationnels interethniques a révélé qu'il n'y a pas de "problème turc "sous-jacent. Au palmarès de l'intégration, les Yougoslaves viennent en tête, car ils entretiennent, dans une forte proportion, des contacts personnels étroits avec leurs collègues de travail allemands et avec des Allemands qui font partie ou non de leur voisinage. Seuls $14 \%$ d'entre eux n'entretiennent aucune relation avec des Allemands. Ce taux est de $16 \%$ chez les Turcs, mais de $27 \%$ (!) chez les Italiens. I.es Turcs sont donc mieux intégrés que les Italiens, tant au niveau des structures (statut professionnel, adhésion, à des associations, assimilation juridique) qu'au nivea u social (relations interethniques), bien que sur le plan de la connaissance des motivations dela maitrise de la langue, de la formation scolaire, du thème du retour. les valeurs enregistrées soient en moyenne plus faibles chez eux qhe chez les migrants italiens (Hill, 1984, p. 165).

\section{I.A QUESTION DE L.A XENOPHOBIE}

Rappelons d'abord qu'isolement ne signifie pas obligatoirement marginalisation. Cette dernière intervient uniquement lorsque, dans leurs efforts d'intégration, les étrangers sont entraînés dans des conflits plus intenses avec les autochtones et qu'en dépit de leurs tentatives pour parvenir au centre, ils sont rejetés à la périphérie. C'est un aspect qui a été souligné par les enquêtes menées sur le niveau de xénophobie de la population allemande (Tsiakalos, 1983 ; Freund, 1983 ; Hoffmann, 1984).

Ces enquêtes on fait apparaître au sein de la population allemande un potentiel de xénophobie susceptible de se manifester dès que certains événements extérieurs ou certaines mises en garde excessives lui en fournissent le prétexte. Conformément à l'hypothèse frustration-agression, les " étrangers " servent facilement de boucs émissaires, surtout quand la situation de l'emploi est en crise. Un sondage représentatif réalisé en 1982 par l'INFAS (Institut des Sciences Sociales Appliquées) indique sans équivoque que les opinions sont beaucoup plus marquées par les émotions que par la connaissance des problèmes, car les attitudes négatives diminuent en fonction de l'intensité des contacts effectifs avec les étrangers et des 
expériences personnelles dont ils ont été l'enjeu. Selon ce sondage, $30 \%$ seulement de la population peuvent être considérés actuellement comme véritablement xénophobes. Au niveau des attitudes et du comportement, les préventions contre les étrangers sont plus nettes dès lors que les personnes interrogées sont plus âgées et que leur niveau scolaire est plus faible.

I a perte massive de sýmpathie qu'enregistrent les étrangers actuellement par rapport au début des années 70 n'a rien d'insolite pour le processus d'intégration. I.e " race-relations-cycle" de Park décrivait déjà que l'" economic welcome " est rapidement suivi d'une recrudescence de l'ethnocentrisme et de l'esprit de compétition et que l'avènement d'une phase de "fair-play" n'est pas automatique mais subordonné à certains facteurs (économiques) secondaires (qui font actuellement défaut).

Il est indéniable qu'une stratification ethnique est en cours en République Fédérale. En moyenne. les étrangers relèvent non seulement de la couche sociale la plus basse de la population allemande. mais en constituent même la frange inférieure. Dans la vie professionnelle et en matière de logement. leur mobilité ascendante est minime. L'augmentation de la durée de séjour n'apporte guère de modifications à leur sort et à la position qu'ils occupent dans l'édifice social, même lorsqu'ils profitent de l'évolution générale des revenus. On ne voit pas encore actuellement comment sortir de l'" ethnic mobility trap " dont parle N. Wiley.

\section{I.E RETOUR AL PAYS I'ORIGINE}

I a situation constamment tendue sur le marché du travail. l'attitude de rejet de la population allemande. les difficultés d'adaptation des étrangers eux-mêmes et. influencée par tous ces facteurs, la politique menée à leur égard. ramenèrent sur le devant de la scène la question du retour.

I.es aides financières au retour proposées actuellement (en fait depuis 1983) n'ont pas encore donné les résultats escomptés. I'une part, la main-d'œuvre spécialisée qu'on aurait préféré garder est repartie : dautre part, ont bénéficié des primes ceux qui avaient de toute façon l'intention de retourner chez eux (effet de "drainage "). Il aurait fallu des moyens financiers beaucoup plus importants pour provoquer une réémigration massive. compte non tenu du fait que, pour beaucoup. la question du retour ne se pose pas pour le moment, même s'ils vivent dans de mauvaises conditions.

Des enquêtes ont été entreprises. dans ce contexte, pour savoir si les étrangers étaient disposés ou non à retourner chez eux. Elles ont montré qu'aujourd hui plus de $80 \%$ de la population turque par exemple, veulent rester en Allemagne ou, du moins, n'ont pas encore fixé la date de leur éventuel retour. Cette ambivalence demande une explication. Voici toute une série de raisons que l'on peut invoquer:

ce qui est déterminant. c'est non seulement le sentiment d'un certain dénuement par rapport à la population allemande. mais aussi la comparaison avec les possibilités d'existence dans le pays d'origine. le retour, s'il se faisait actuellement, s'accompagnerait pour nombre de travailleurs immigrés d'un important manque à gagner. qu'ils sont à un niveau de salaire relativement élevé et à une couverture sociale d'une ampleur le plus souvent inconnue chez eux. A cela s'ajoute 
l'incertitude de retrouver un emploi dans le pays d'origine, car il est fréquent que la situation ne se soit pas sensiblement modifiée pendant toute la durée de l'émigration.

Sur ce point, les travailleurs indépendants se montrent plus satisfaits que les salariés. En effet, après de longues années d'absence, ceux-ci ont fréquemment bien des difficultés à reprendre pied dans ce réseau d'intermédiaires et de relations qu'on appelle " circuit ", "giro ", où tout se fait par contacts personnels (Grassi, 1980; Körner Werth, 1981). C'est pourquoi, une fois arrivés, ils sont étonnamment nombreux à vouloir repartir, d'autant que, pour leurs pays d'origine, le retour offre peu d'intérêt économique parlant (Rhoades. 1981 ; Ralle, 1981).

-. Il semble qu'en période de crise. la propension au retour soit plus marquée dans la population rurale que dans la population urbaine, celle-ci s'accommodant mieux des incertitudes de l'avenir dans le pays d'accueil, surtout si les familles se sont regroupées. L a faculté d'absorption des zones rurales et leurs débouchés inspirent davantage confiance (Kallweit Kudat, 1976), surtout chez les migrants d'un certain âge (Bernitt, 1981, p. 135).

Abstraction faite des cas où les migrants anticipent sensiblement sur les difficultés qu'ils auraient à surmonter au cas où ils souhaiteraient un jour repartir à l'étranger après le retour au pays (Herrmanns Lienau, 1979), on peut dire qu'en plus des raisons déjà citées, l'éducation bi-culturelle des enfants freine beaucoup les retours (Harder, 1980). I.es liens de la deuxième et de la troisième génération avec le pays d'origine sont souvent devenus si ténus que celui-ci leur semble plus lointain que le pays d'accueil (connaissance de la langue, système scolaire, coutumes, etc.). En réalité, quand les parents décident de rentrer, c'est souvent parce que les enfants en manifestent le désir et l'on ne sait pas encore très bien aujourd'hui quels peuvent être les effets d'une éducation bi-culturelle.

Toutes ces raisons mises à part, pour les deux-tiers des migrants le problème de la réémigration ne se pose pas, du simple fait qu'ils bénéficient, en tant que ressortissants de la CEE, de la liberté de circulation et d'installation garanties par le droit communautaire. Etant donné par ailleurs que la " mise à la porte " de tous les autres ne serait supportable ni sur le plan économique ni sur le plan culturel, les hommes politiques n'ont au contraire d'autre alternative que de se préparer à voir le nombre de résidents étrangers plutôt s'accroître dans les années qui viennent.

En effet, la connaissance des mouvements conjoncturels enseigne qu'en période d'essor économique, il faut s'attendre à l'arrivée de nouveaux migrants et que leur nombre atteint alors un niveau généralement plus élevé que le niveau initial. De son côté, la politique communautaire, qui vise à élargir l'éventail des possibilités migratoires, ne fera qu'accentuer la tendance existante (d'autant plus que l'arrêt du recrutement, toujours en vigueur, ne s'applique pas aux pays de la Communauté).

Mais le facteur d'amplification le plus important est le regroupement des familles garanti jusqu'ici par toutes les politiques. Actuellement, la règle est que les étrangers peuvent faire venir en République Fédérale leurs conjoints et leurs enfants de moins de 16 ans (de moins de 21 ans pour les ressortissants de la CEE). On estime que ce potentiel migratoire est de l'ordre de 800000 à 1000000 de 
personnes, de sorte que la population étrangère pourrait augmenter de 2400000 d'ici la fin du siècle et qu'elle compterait 7 millions de personnes en l'an 2000 (Bade, 1983, p. 106).

Tout cela démontre, on ne peut plus pertinemment, qu'on ne résoudra pas le problème des étrangers par une "immigration à rebours ". L'alternative est simple : ou bien on le remet à plus tard et son potentiel conflictuel en sera accru d'autant (Koch Arzberger, 1985, p. 187) ou bien il doit être résolu dès maintenant, et en République Fédérale. Mais pour cela, il faut entreprendre d'autres recherches ayant pour objet de savoir non seulement combien de temps les étrangers prévoient de rester en Allemagne, mais aussi comment ils envisagent leur vie dans ce pays.

\section{STRATEGIES DE RECHERCHE SOUHAITABLES POUR L'A VENIR}

Malgré l'ampleur du champ ouvert en Allemagne depuis 1972 (deuxième phase) et surtout depuis 1979 (troisième phase) à la recherche sociologique sur la question des travailleurs, immigrés et malgré l'abondance des données collectées, la théorie du phénomène migratoire a singulièrement peu progressé. On a globalement l'impression qu'elle ne s'est pas encore totalement libérée de l'optique réductrice du début et que c'est encore le point de vue du pays d'accueil qui prévaut. Mais ces immigrés, qui sont-ils : Comment vivent-ils ? Quels sont les registres sur lesquels ils s'expriment? Quelle est leur perspective culturelle propre ? Quelles stratégies développent-ils dans leurs contacts avec l'univers étranger du pays d'accueil ? Autant d'interrogations qui n'ont reçu que des ébauches de réponses quand toutefois elles ont retenu l'attention. C'est pourquoi il ne nous semble pas abusif de constater que d'importants aspects de la vie des migrants échappent encore à notre compréhension après une génération de recherches.

Un pas important serait franchi si l'on changeait de perspective sur trois points, si l'on envisageait le phénomène migratoire en s'attachant à l'interpréter, à en étudier la dynamique et les interactions.

\section{LA MIGRATION VUE PAR LES MIGRANTS}

Savoir comment les migrants se comportent et agissent, nous devons d'abord faire abstraction de notre manière de voir le "phénomène étranger ", ou plutôt apprendre dans un premier temps à mieux cerner les structures de perception des étrangers en question et se demander comment ils ressentent l'univers dans lequel ils vivent : comme quelque chose d'étrange, qui les rejette, qui les provoque? (Dietsche, 1984).

Si nous nous en tenons uniquement à nos besoins économiques et politiques, il ne sera guère possible de corriger les insuffisances de la recherche. Il est nécessaire, non seulement d'intensifier les investigations, mais aussi de les concevoir différemment. ce qui signifie qu'elles doivent être également placées sous le signe de la sociologie de la culture. Mais il ne suffit pas, là non plus, de constater que les traditions et les valeurs culturelles sont différentes. Nous sommes bien loin de connaître en détail ces valeurs (Kakaletris, 1984). 
Shanin a déjà donné quelques indications sur ce point. Il a montré que la plupart des travailleurs immigrés sont des paysans qui abordent les sociétés industrialisées des pays d'accueil avec les habitudes mentales qui leur viennent de sociétés paysannes. Une partie de leurs conflits et de notre incompréhension tient sans doute au fait que nous connaissons mal ce týpe de société où s'appliquent des comportements qui diffèrent des nôtres jusque dans les stratégies mises en ouvre pour combattre les maladies (Shanin, 1980: Zimmermann, 1985).

I a situation de la recherche se complique encore du fait que nous ne savons pas en quelles circonstances sont activées ou réactivées des valeurs et quelles sont ces valeurs. I e phénomène est particulièrement sensible dans le domaine de l'éducation, comme l'a montré l'exemple des Turcs (" returquisation "). la situation est analogue dans le domaine de la langue ("refus linguistique "), du comportement relationnel qui présente des caractères différents selon que l'on a affaire à telle ou telle population, etc. Sur ce point, la différence est frappante entre les Italiens d'une part et les Yougoslaves et les Grees de lautre. Et nous en ignorons encore les raisons.

Il est également important de distinguer les modèles de pensée et de comportement des hommes et des femmes et bien plus encore de la première et de la seconde génération. I à aussi nous savons encore trop peu de choses sur la constitution de " niches " culturelles. Quels sont les gagnants, quels sont les perdants de la migration. quels sont ceux qui la subissent avec résignation ? Car les avantages et les inconvénients de la migration ne sont pas les mêmes pour tout le monde. Dans ce domaine. la recherche, telle qu'elle a été pratiquée jusqu'aujourd'hui, n'a pas suffisamment différencié ses objectifs. Nous nous sommes souvent contentés de constater le choc des cultures, mais nous savons encore trop peu de choses sur les besoins refoulés, les comportements d'adaptation, l'alternative d'intégration et motivation au retour. le pouvoir de la mémoire et de l'imagination, etc.

I es enquêtes consacrées aux voux "exprimés " sont ici de peu de secours. Schiffauer a montré dans ses travaux (1983, 1984) quelle intensité doivent avoir les entretiens si l'on veut parvenir à cerner les caractères spécifiques d'une perception. les déclencheurs de comportements, les stratégies à long terme, sans doute parce que les personnes interrogées elles-mêmes ne se les formulent pas clairement. La seule voie qui permettra de progresser en ce domaine, si tant est que cela soit possible, sera de poursuivre linvestigation sur la base d'une exploration qualitative, d'autant plus qu'en matière de contacts interculturels, il est parfaitement impossible d'avoir un comportement "aseptisé " lorsqu'on enregistre des données (Allerbeck Hoag, 1985, p. 246).

\section{LINTEGRATION COMPRISE COMME UN PROCESSUS}

L.es programmes des partis, les propos des hommes politiques et les déclarations des grandes organisations patronales et ouvrières, révèlent une curieuse valsehésitation entre les appels à l'intégration et les garanties d'authenticité, le respect de l'enracinement des migrants dans leur tradition et la tentation simultanée de n'en pas tenir compte. Il n'y a là rien d'étonnant, car on ne sait pas encore aujourd'hui ce qu'il faut entendre par " intégration ". Pour définir celle-ci, il ne suffit pas d'énon- 
cer une volonté politique, il faut aussi prendre en compte ce qui est politiquement réalisable. Cela implique donc que l'on sache également ce que les étrangers euxmêmes entendent par " intégration". Quelle intégration sont-ils en mesure ou veulent-ils consciemment ou inconsciemment assumer - si tant est qu'ils la désirent ? Ce sont là des composantes qu'il est difficile d'éliminer comme l'indique une angoisse largement répandue chez les étrangers, l'« angoisse de déculturation " qui peut s'emparer d'eux quand ils sont confrontés aux exigences de l'intégration.

Le temps est assurément un facteur qui joue un rôle important. La longueur du séjour atténue sans doute quelques-unes de ces angoisses et de ces résistances. Peut-elle cependant contrebalancer le poids des liens inconscients à la tradition? Nous l'ignorons. Sur ce point, la recherche n'a encore rien pu apporter, sa conception du temps étant vraisemblablement trop unidimensionnelle. A cela vient s'ajouter que la plupart des " offres " d'intégration masquent, semble-t-il, un " race-relations-cycle " - de 4 phases successives comprenant " contact ", " compétition ", " accommodation ", " assimilation" (Park, 1950, p. 150 ; Gordon, 1968).

Quels que soient les modèles auxquels on se rallie, qu'il s'agisse de ceux dont nous venons de parler ou de modèles similaires, ce qui frappe, c'est l'absence de représentation permettant d'appréhender, sous une forme réellement différenciée, le déroulement de la démarche d'intégration. La plupart du temps, on aborde le processus en le schématisant, sans se demander, qui prend part à cette évolution, dans quelle proportion et à quelle occasion ; à quel moment se termine le processus d'acquisition et quand on peut, par conséquent, considérer que le cycle est accompli ; quelle est la génération où l'on peut s'attendre à constater la fin du cycle. Si enfin ce processus relève d'une évolution purement linéaire ou s'il faut imaginer tout un éventail d'influences diverses qui feront de la dernière phase d'assimilation un événement rare.

C'est la raison pour laquelle Hettlage/Hettlage (1984, p. 375 et suiv.) proposent de concevoir l'intégration comme un processus d'identification dont on pourrait cerner la complexité par la notion d'" univers intermédiaire ". Etant donné que le processus de recherche identitaire passe par la nécessité de trouver une unité entre deux cultures, cette unité ne peut être envisagée que comme une démarche qui, compte tenu du fonds d'expériences hérité de la tradition et des influences du moment, tente de constituer des ensembles intégrant des éléments empruntés aux deux univers. Ces ensembles peuvent se modifier avec le temps (on parlera d'" univers intermédiaires " à contenu d'intégration variable). La notion préconisée permet également de rendre compte des blocages durables que peut engendrer l'intégration, blocages qui renforcent à leur tour la ségrégation, l'isolement, l'apathie, difficiles à vaincre en l'espace d'une génération.

Il y aurait ici matière à plus amples recherches sur les spécificités d'attitudes des populations immigrées : les facteurs culturels, l'expérience acquise, le poids des traditions n'ont pas la même influence sur tous. Seule la connaissance des facteurs qui s'exercent sur les populations de migrants nous permettra de dire si telle ou telle politique est réaliste, si telle ou telle manière de concevoir l'intégration est opportune ou encore de définir le contenu notionnel de l'» intégration " dans un contexte pluriculturel. 


\section{LES INTERACTIONS : LE « DRAME " ENTRE LE PAYS D'ACCUEIL ET LES TRAVAILLEURS IMMIGRES}

On ne saurait se contenter d'expliquer la méthode d'étude du processus aux seuls migrants et à leurs stratégies d'assimilation. Comme ceux-ci ont acquis une partie de leur expérience dans le pays d'accueil, il est nécessaire d'inclure celui-ci dans l'analyse, car il entre dans le jeu des interactions. Le terme de "drame " a une double signification : il implique, premièrement, qu'on fonctionne sur des registres différents et qu'on donne de soi une image différente quand on est pris dans le choc de deux cultures (Goffman, 1983, p. 31 et suiv.), deuxièmement, que les intentions manifestées alors sont souvent incomprises et mènent à un dialogue de sourds. Cette vue "dramatique " des choses (Lipp, 1984, p. 8 et suiv.) a son importance dans la question des migrations si l'on veut mieux analyser les hésitations, les blocages et les ruptures du processus d'intégration. Souvent, en effet, il s'avère que ces situations masquent des déceptions et des frustrations : les étrangers se sont adressés au pays d'accueil en faisant appel aux ressources qu'ils avaient coutume d'utiliser dans l'action et, incompris de lui, ils se retirent à distance, dans l'expectative. Ces blessures conduisent souvent à un type d'univers intermédiaire qui rend fort difficile tout rapprochement ultérieur. Inversement, le pays d'accueil peut ressentir comme une provocation la distance adoptée par les étrangers et vouloir la sanctionner par le mépris.

Dans le meilleur des cas, le processus de rapprochement mutuel se déroule sans drame lorsque les deux parties se trouvent dans la même phase d'ouverture l'une par rapport à l'autre. Mais le cas le plus fréquent est celui d'un déphasage chronologique des attitudes de distanciation et de rapprochement, décalage qui fait obstacle à la communication.

Ces phénomènes ne manquent pas d'intérêt quand on les rapporte par exemple à la xénophobie des autochtones ou encore à l'angoisse de déculturation des étrangers. Il ne suffit pas de constater que l'intensification des contacts entraîne une résorption des craintes mutuelles. Chez les uns et les autres, la prise de contact et la propension à communiquer doivent en outre se situer dans une phase d'évolution congruente (Hettlage, 1986). Les rapports entre les migrants et leur pays d'origine peuvent s'interpréter à l'aide des mêmes critères. On remarquera sur ce point que l'émigration s'accompagne souvent de blessures dramatiques dues au déracinement ou à de secrètes rancunes contre le pays d'origine ; intériorisées par le migrant, elles seront un traumatisme pour le restant de ses jours, mais lui donneront aussi le sentiment paradoxal d'être plus étroitement solidaire de son pays.

Là encore, cette manière de penser et de se comporter a un aspect théâtral. Par ses attaches, dans son désir de s'affirmer et d'accéder à un statut, le travailleur immigré demeure lié au retour dans son pays sur le plan émotionnel et social. Mais souvent vient le jour où il constate, à son corps défendant, que le pays qui l'a autorisé à partir n'est pas tellement très intéressé à son retour parce qu'il n'a toujours rien à lui proposer. Tant que persisteront ces attaches, il est probable que l'immigré ne saisira aucune chance réelle d'examiner sérieusement les " offres d'intégration " du " pays d'accueil ». Cela peut amener la société d'accueil à reprendre ses distances et déclencher une nouvelle phase d'incongruence dans le drame relationnel où l'un et l'autre sont engagés. 
Une recherche centrée sur les interactions pourrait apporter une contribution non négligeable à la compréhension des problèmes migratoires, communauté par communauté, pour peu qu'elle définisse pour chacune d'elles, où se situent et comment s'élaborent ces univers communs ou distincts. Les premiers jalons sont posés. Mais sur la voie ouverte par ces trois orientations de recherche, nous n'en sommes qu'à nos premiers pas. Si nous persévérons, une quatrième phase, décisive celle-là, pourrait bien être inaugurée par la recherche allemande sur les migrations.

La traduction française du texte de R. Hettlage a été effectuée par François Kihm, Maître de Conférences d'Allemand à l'Université de Poitiers.

\section{Note et références bibliographiques}

(1) A ce sujet, les Suisses font moins de sentiment et occultent moins leur position sur la question. Ils parlent franchement de Fremdarbeiter, terme que l'on réprouve en Allemagne (N.d.T. : les Fremdarbeiter sont littéralement des "travailleurs qui ne sont pas d'ici").

AKPINAR (U). Zur Schulsituation des Kinder ausländischer Arbeitnehmer, (La situation scolaire des enfants des travailleurs étrangers), dans : Langenohl-Weyer et coauteurs. Zur Integration der Ausländer im Bildungsbereich, l'intégration des étrangers dans le système éducatif. Munich, 1979.

ALBRECHT (G.). Soziologie der geographischen Mobilität. Zugleich ein Beitrag zur Soziologie des sozialen Wandels, (Sociologie de la mobilité géographique. Contribution à la sociologie du changement social). Stuttgart, 1972.

ALBRECHT (P.A.) et PFEIFFER (Ch.). Die Kriminalisierung junger Ausländer. Befunde und Reaktionen sozialer Kontrollinstanzen, (Les facteurs de la délinquance chez les jeunes étrangers. Inventaire et réactions des instances de contrôle social). Munich, 1979.

ALLERBECK (K.R.) et HOAG (W.J.). Wenn Deutsche Ausländer befragen. Ein Bericht über methodische Probleme und praktische Erfahrungen. (Quand des Allemands interrogent des étrangers. Questions de méthodes et expérience pratique), dans : Zeitschrift für Soziologie, Revue de sociologie, 14 (1985) 3, pp. 241246.

BADE (K.). Vom Auswanderungsland zum Einwanderungsland? Deutschland 1880-1980, (L'Allemagne de 1880 à 1980 : un pays d'émigration devenu pays d'immigration ?). Berlin, 1983.

BA YAZ (A.) (édit.). Integration. Anpassung an die Deutschen ?, (L'intégration consiste-t-elle à s'adapter aux Allemands ?). Weinheim, 1984.

BERNITT (M.). Die Rückwanderung spanischer Gastarbeiter. Der Fall Andalusien, (Le retour au pays des travailleurs immigrés espagnols. Le cas de l'Andalousie). Königstein/Ts.

BIELEFELD (U.), KREISSL (R.) et MUNSTER (Th.). Junge Ausländer im Konflikt, (Jeunes étrangers en conflit). Munich, 1982.

BINGEMER (K.), MEISTERMANN-SEEGER (E.) et NEUBERT (E.) (édit.). Leben als Gastarbeiter. Geglückte oder miBglückte Integration, (La vie de travailleưr immigré. Succès ou échec de l'intégration). Cologne/Opladen, 1970.

BIRKENFELD (H.) (édit.). Gastarbeiterkinder aus der Türkei. Zwischen Eingliederung und Rückkehr, (Les enfants des travailleurs immigrés turcs. Entre intégration et retour). Munich, 1982.

BOOS-NUNNING (U.), HOHMANN (M.) et REICH (H.H.). Integration ausländischer Arbeitnehmer. Schulbildung ausländischer Kinder, (L'intégration des travailleurs étrangers. Formation scolaire des enfants d'étrangers). Bonn, 1976.

BOR RIS (M.). Ausländische Arbeiter in einer GroRstadt, (Ouvriers étrangers dans une grande métropole). Francfort, 1973. 
BUNDESANSTALT FUR ARBEIT (Office Fédéral du Travail, édit.). Ausländische Arbeitnehmer. Beschäftigung, Anwerbung, Vermittlung. Einführungsbericht 1969. Ergebnisse der Repräsentativ-untersuchung vom Herbst 1968. (Travailleurs étrangers. Emploi, recrutement, placement. Rapport d'introduction de 1969. Résultats du sondage représentatif effectué en automne 1968). 1970.

BUNDESANSTALT FUR ARBEIT (édit.). Repräsentativuntersuchung 72. Beschäftigung ausländischer Arbeitnehmer. (Sondage représentatif de 1972. L'emploi des travailleurs étrangers). Nuremberg, 1973.

BUNDESJUGENDKURATORIUM (Conseil Fédéral de la Jeunesse). Zur Situation ausländischer Kinder une Jugendlicher in der Bundesrepublik. (La situation des enfants et des adolescents étrangers en République Fédérale). Bonn. 1976.

DIETSCHE (P.). Das Erstaunen üher das Fremde, (Etrangeté qui nous étonne). Francfort, 1984.

DIRICKS (Y.) et KUDAT (A.). Ghettos : Individual or systemic Choice ? Berlin, 1975.

DOHSE (K.). Ausländerpolitik und betriebliche Ausländerdiskriminierung. (Politique à l'égard des étrangers et discrimination dans l'entreprise, dans : Leviathan), 1981, 3-4, pp. 4999-526.

ELWERT (G.). Probleme der Ausländerintegration. Gesellschaftliche Integration durch Binnenintegration ?. (Les problèmes de l'intégration des étrangers. L'intégration sociale doit-elle passer par l'intégration endogène ?) dans : Kölner Zeitschrift für Soziologie und Sozialpsychologie, Revue de sociologie et de psychologie sociale de Cologne, 34 (1982), pp. 717-731.

ESSER (H.). Aspekte der Wanderungssoziologie. Assimilation und Integration von Wanderern, ethnischen Gruppen und Minderheiten, (Aspects de la sociologie des migrations. L'assimilation et l'intégration de migrants, de groupes et de minorités ethniques). Darmstadt, 1980.

DEUTSCHES JUGENDINSTITUT (Institut Allemand de la Jeunesse, édit.). Sozialisationsbedingungen ausländischer Kinder und Jugendlicher in der Bundesrepublik, (Les conditions de socialisation des enfants et des adolescents étrangers en République Fédérale). Munich, 1980.

FREUND (W.S.) (édit.). Gastarbeiter. Integration oder Rückkehr? Grundfragen der Ausländerpolitik, (Les travailleurs immigrés. Intégration ou retour au pays? Questions fondamentales de la politique à l'égard des étrangers). Neustadt/ WeinstraBe, 1980.

GLIEDNER-SIMON (A.). Ausländer zwischen Integration und Remigration. Eine Dokumentation von Forschungsarbeiten und Literatur der Jahre 1982-1985. (Les étrangers entre l'intégration et la réémigration. Documentation rassemblant travaux de recherche et publications des années 1982-1985). Bonn, 1986.

GOKALP (A.). Migrants' Children in Western Europe : differential socialization and multicultural problems, dans : International Social Science Journal, 36 (1984) 3, pp. 487-500.

GOFFMAN (E.). Wir alle spielen Theater. Die Selbstdarstellungen im Alltag. (Nous jouons tous la comédie. Les images que nous donnons de nous-mêmes dans la vie quotidienne). Munich/Zurich, $1983^{4}$.

GORDON (M.). Assimilation in American Life. The Role of Race, Religion and National Origin. New York, 1964, 19682.

GRASSI (M.). Die Arbeitsmigration im italienischen Süden und die gesellschaftliche Problematik der Reintegration. (La migration de population active en Italie méridionale et le problème social de la réintégration). Berlin, 1980.

HAAS (P.), GRUGELKE (G) et coauteurs. Kinder ausländischer Arbeitnehmer im schulischen und auBerschulischen Bereich. (Les enfants des travailleurs étrangers dans le domaine scolaire et extra-scolaire). Berlin. 1980.

HARDER (E.). Rückkehr oder Verbleib. Eine Befragung griechischer Arbeiter in Nordrhein-Westfalen. (Retourner au pays ou rester en RFA ? Résultats d'une enquête menée auprès d'ouvriers grecs en Rhénanie du Nord-Westphalie). Melle, 1980.

HECKMANN (F.). Einwanderung als ProzeR, Limmigration considérée comme processus, dans : BLASCHKE (J.), GREUSSING (K) (édit.). "Dritte Welt " in Europa. Probleme der Arbeitsimmigration. Le “Tiers-Monde " en Europe. (Les problèmes de l'immigration active). Francfort, 1980, pp. 95-124.

HECKMANN (F.). Die Bundesrepublik, ein Einwanderungsland? Zur Soziologie der Gastarbeiterbevölkerung als Einwanderungsminorität, (La République Fédérale est-elle un pays d'immigration ? Contribution à la sociologie des populations de travailleurs étrangers considérées comme minorités issues de l’immigration). Stuttgart, 1981. 
HERMANNS (H.) et LIENAU (C.). Rückwanderung griechischer Gastarbeiter und Entwicklung ländlicher Räume in Griechenland. (Le retour des travailleurs immigrés grecs et le développement des espaces ruraux en Grèce), dans : WEBER (P.) (édit.). Periphere Räume, Espaces périphériques, Paderborn, 1979, pp. 53-86.

HETTLAGE (R.). Der Fremde : Kulturmittler, Kulturbringer, Herausforderer von Kultur, (L'étranger médiateur, vecteur et instigateur de culture), dans : LIPP (W.) (édit.). Kulturı̀pen. Types culturels. Berlin, 1986 (à paraître).

* Titre exact : Kulturtypen, Kulturcharaktere - Träger. Mittler und Stifter von Kultur. Types et caractères culturels : supports, médiateurs et fondateurs de culture.

HETTLAGE-VARJAS (A.) et HETTLAGE (R.). Kulturelle Zwischenwelten. Fremdarbeiter-eine Ethnie?, Univers culturels intermédiaires. (Les travailleurs étrangers constituent-ils une ethnie ?) dans : Schw'eizerische Zeitschrift für Soziologie, Revue suisse de sociologie, 1984, 2, pp. 357-404.

HILL (P.B.). Determinanten der Eingliederung von Arbeitsmigranten, (Les facteurs déterminants de l'intégration des migrants actifs). Königstein/Ts., 1984.

HOFFMANN (L.). Soziologie der Ausländerfeindlichkeit, (Sociologie de la xénophobie). Weinheim/Bâle, 1984.

HOFFMANN-NOWOTNY (H.J.). Migration. Ein Beitrag zu einer soziologischen Erklärung, (La migration. Contribution à une explication sociologique du phénomène). Stuttgart, 1970.

HOFFMANN-NOWOTNY (H.J.) et HONDRICH (K.O.) (édit.). Ausländer in der Bundesrepublik Deutschland und in der Schweiz. Segregation und Integration. Eine vergleichende Untersuchung. (Les étrangers en République Fédérale d'Allemagne et en Suisse. Ségrégation et intégration. Etude comparative). Francfort/New York, 1982.

HOFFMEYER-ZLOTNIK (J.). Gastarheiter im Sanierungsgebiet. Beiträge zur Stadtforschung. (Les travailleurs immigrés dans les quartiers en voie de rénovation. Contributions à la recherche en matière d'urbanisme). Hambourg, 1977.

Institut für angewandte Sozialwissenschaft (Institut des sciences sociales appliquées), INFAS : Meinungen und Einstellungen zum Ausländerproblem. Endbericht und Tabellenband, (Opinions et attitudes face au problème des étrangers. Rapport de conclusion et tableaux). Bonn-Bad Godesberg, 1982.

IPSEN (D.). Aufenthaltsdauer und Integration ausländischer Arbeiter. (Durée de séjour et intégration des travailleurs étrangers) dans : Zeitschrift für Soziologie. Revue de sociologie, 6 (1977) 4, pp. $403-424$.

IPSEN (D.). Wohnsituation und Wohninteresse ausländischer Arbeiter in der Bundesrepublik Deutschland. (Situation et aspirations des travailleurs étrangers en RFA en matière de logement), dans : Leviathan, 6 (1978) 4, pp. 558-573.

KAKALETRIS (G.). Kulturelle Probleme der griechischen Familie in der BRD und der Rolle der Kirche, (Problèmes culturels de la famille grecque en RFA. Le rôle de l'Eglise). Tübingen, 1984.

KALLWEIT (H.) et KUDAT (A.). Rückwanderung ausländischer Arbeiter : zwangsweise oder freiwillig ? Pilotsıudie über soziale Merkmale türkischer Rückwanderer. (Le retour des travailleurs étrangers dans leurs pays d'origine : contrainte ou liberté ? Etude pilote des caractéristiques sociales des Turcs qui retournent dans leur pays). Berlin, 1976.

KISKER (K.P.). Wirtschaftliche und soziale Hintergründe der Situation ausländischer Arbeitnehmer, (L'arrière-plan économique et social de la situation des travailleurs étrangers), dans: HANSEN (G.), KLEMM (K.) (édit.). Kinder ausländischer Arbeiter, (Les enfants des ouvriers étrangers). Bochum, 1979. pp. II-29.

KORNER (H.), WERTH (M.) (édit.). Rückw'anderung und Reintegration von ausländischen Arbeitnehmern in Europa. (Retour et réintégration des travailleurs étrangers en Europe). Sarrebruck, 1981.

KOCH-ARZBERGER (C.). Die schwierige Integration. Die bundesrepublikanische Gesellschaft und ihre 5 Millionen Ausländer, (La difficile intégration. La société ouest-allemande et ses 5 millions d'étrangers). Opladen, 1985.

KREMER (M.) et SPANGENBERG (H.). Assimilation ausländischer Arbeitnehmer in der Bundesrepublik Deutschland, (L'assimilation des travailleurs étrangers en République Fédérale d'Allemagne). Königstein/Ts.

KUDAT (A.). Stability and change in the Turkisch Family at Home and Abroad : Comparative Perspectives. Berlin, 1975. 
KURZ (U.). Partielle Anpassung und Kulturkonflikt. Eine soziologische Untersuchung der Gruppenstruktur und des Anpassungsverhaltens in einem italienischen Arbeiterlager Münchens, (Adaptation partielle et conflit de cultures. Etude de la structure de groupe et du comportement d'adaptation dans un camp de travailleurs italiens de Munich), dans : Kölner Zeitschrift für Soziologie und Sozialpsychologie. Revue de sociologie et de psychologie sociale de Cologne, 7 (1965), pp. 814-832.

LEBON (A.) et FALCHI (G.). New Developments in Intra-European Migration. Since 1984, dans : International Migration Review, 14 (1980) 4 pp. 539-579.

LEITNER (G.). Gastarbeiter in der städtischen Gesellschaft. Segregation, Integration und Assimilation von Arbeitsmigranten. Am Beispiel jugoslawischer Gastarbeiter in Wien, (Les travailleurs immigrés dans la société urbaine. Ségrégation, intégration et assimilation des migrants actifs : l'exemple des travailleurs immigrés yougoslaves à Vienne). Francfort/New York, 1983.

LEY (K.). Frauen in der Emigration. Eine soziologische Untersuchung der Lebens - und Arbeitsbedingungen italienischer Frauen in der Schweiz, (Les femmes dans l'émigration. Etude sociologique des conditions de vie et de travail des Italiennes en Suisse). Frauenfeld, 1979.

LOHRMANN (R.) et MANFRASS (K.) (édit.). Ausländerbeschäftigung und internationale Politik. (l'emploi de main-d'œuvre étrangère et la politique internationale). Munich, 1974.

LIPP (W.). Kultur dramatologisch, (La culture dans ses aspects dramatologiques) dans : Osterreichische Zeitschrift für Sociologie, Revue autrichienne de sociologie, 9 (1984) 1-2, pp. 8-25.

LIGURAS (S.). Familien zwischen zwei Kulturen. Eine Untersuchung zum soziokulturellen Wandel griechischer Familien in der Bundesrepublik Deutschland, (Familles entre deux cultures. Etude du changement culturel dans des familles grecques vivant en République Fédérale d’Allemagne). Francfort, 1981.

McRAE (V.). Die Gastarbeiter. Daten, Fakten, Probleme, (Les travailleurs immigrés. Données, faits et problèmes). Munich, $1981^{2}$.

MERTENS (G.) et AKPINAR (U.). Türkische Migrantenfamilien. Familienstrukturen in der Türkei und in der Bundesrepublik. Ausgleichungsprobleme türkischer Arbeiterfamilien : Beispiel Westberlin, (Les familles de migrants turcs. Structures familiales en Turquie et en République Fédérale. Difficultés des familles ouvrières turques à la recherche d'un équilibre. Données recueillies à Berlin-Ouest). Bonn, 1977.

MEHRLANDER (U.). Beschäftigung ausländischer Arbeitnehmer in der Bundesrepublik Deutschland unter spezieller Berücksichtigung von Nordrhein-Westfalen, (L'emploi des travailleurs étrangers en République Fédérale d'Allemagne et tout particulièrement en Rhénanie du Nord-Westphalie). Opladen, $1969,1972^{2}$.

MEHRLANDER (U.). Soziale Aspekte der Ausländerbeschäftigung, (Aspects sociaux de l'emploi de maind'œuvre étrangère). Bonn/Bad Godesberg, 1974.

MUNSCHER (A.). Ausländische Familien in der Bundesrepublik Deutschland. Familiennachzug und generatives Verhalten, (Familles étrangères en République Fédérale d'Allemagne. Regroupement familial et natalité). Munich, 1979.

NELSON (B.). Der Ursprung der Moderne. Vergleichende Studien zum Zivilisationsprozess, (Les origines de l'époque moderne. Etudes comparées du processus de civilisation). Francfort, 1977.

NEUMANN (U.). Erziehung ausländischer Kinder. Erziehungsziele und Bildungsvorstellungen in türkischen Arbeiterfamilien, (L'éducation des enfants d'étrangers. Comment les familles ouvrières turques conçoivent les objectifs de l'éducation et de la formation de leurs enfants). Düsseldorf, 1980.

PAPAIOANNU (S.). Arbeitsorientierung und GesellschaftsbewuRtsein von Gastarbeitern in der Bundesrepublik, (Recherche d'un emploi et conscience sociale chez les travailleurs immigrés vivant en République Fédérale). Francfort/Berne, 1983.

PAPALEKAS (J.-Chr.) (édit.). Die Ausländerfrage. Gastarbeiter im Spannungsfeld von Integration und Reintegration, (La question des étrangers. Les travailleurs immigrés partagés entre intégration et réintégration). Herford, 1983.

PARK (R.E.) et MILLER (H.A.). Old-World Traits Transplanted. New York, $1921,1969$.

PARK (R.E.). Race and Culture. New York, 1950.

PETERS (A.). Ausländische Arbeitnehmer. Literatur und Forschungsprojekte, (Travailleurs étrangers. Publications et projets de recherche). Nuremberg, 1982. 
RALLE (B.). Modernisierung und Integration am Beispiel der Türkei, (Modernisation et intégration. L'exemple de la Turquie). Sarrebruck, 1981.

REIMANN (H.). Anmerkungen zur Gastarbeiterpolitik. Insulation - ein neus Integrationskonzept?. (Remarques sur la politique pratiquée envers les travailleurs immigrés. L'insularisation, une nouvelle manière de concevoir l'intégration ?) dans : BLUM (R.) et STEINER (M.) (édit.). Aktuelle Probleme der Marktwirtschaft in gesamt- und einzelwirtschaftlicher Sicht, (Problèmes actuels de l'économie de marché du point de vue micro- et macroéconomique). Berlin, 1984, pp. 65-85.

REIMANN (H.). Labor Inporting Countries : Federal Republic of Germany, dans : KRANE (R.E.) (édit.) : International Labor Migration in Europe. New York, pp. 63-87.

RENNER (E.). Erziehungs- und Sozialisationsbedingungen türkischer Kinder. Ein Vergleich zwischen Deutschland und der Türkei, (Les conditions d'éducation et de socialisation des enfants turcs. Comparaison entre l'Allemagne et la Turquie). Neuburgweiher, 1975.

RHOADES (V.K.) et RHOADES (R.E.). Rückwandereung und Entwicklung im Agrarsektor. Beispiele aus Granada, (Réémigration et développement agricole. L'exemple de Grenade) dans : Sociologicus 31 (1981) 1, pp. $38-60$.

ROSEN (R.) et STUVE (G.). Ausländische Mädchen in der Bundesrepublik, (Jeunes filles étrangères en République Fédérale). Munich, 1985.

SASSEN (C.). Die Auswirkungen des Kulturwechsels auf Selbstkonzept und Schulleistung. Eine empirische Untersuchung mit italienischen Familien in der BRD, (Les répercussions du changement de culture sur l'idée de soi et les résultats scolaires. Enquête empirique menée auprès de familles italiennes en RFA). Cologne, 1984.

SCHIFFAUER (W.). Die Gewalt der Ehre. Erklärungen zu einem türkisch-deutschen Sexualkonglikt, (La puissance du sentiment de l'honneur. Commentaire d'un délit sexuel opposant Turcs et Allemands). Francfort, 1983.

SCHIFFAUER (W.). Religion und Identität. Eine Fallstudie zum Problem der Reislamisierung bei Arbeitsmigranten, (Religion et identité. Etude de cas concernant le problème de la "réislamisation " des migrants actifs), dans : Zw'ischenwelten der Gastarbeiter. Sondernummer der Schweizerischen Zeitschrift für Soziologie, (Les univers intermédiaires des travailleurs immigrés). Numéro spécial de la Revue suisse de sociologie, 10 (1984) 2, pp. 485-516.

SCHIERUP (C.U.). The Immigrants and the Crisis, dans : Acta Sociologica 28 (1985) 1, pp. 21-33.

SCHILDMEIER (A.). Integration und Wohnen, Intégration et habitat. Hambourg, 1975.

SHANIN (T.). Die Bauern kommen. Migranten, die arbeiten, Bauern, die reisen und Marxisten, die schreiben. (Les paysans arrivent. Des migrants qui travaillent, des paysans qui voyagent et des marxistes qui publient), dans : BLASCHKE (J.) et GEUSSING (K.) (édit.) : "Dritte Welt" in Europa. Probleme der Arbeitsimmigration, (Le "Tiers-Monde " en Europe. Problèmes de l'immigration active). Francfort, 1980, pp. $72-85$.

SIMMEL (G.). Exkurs über den Fremden, (Essai sur l'étranger), dans, du même auteur : Soziologie, Berlin, 1968.

SPIES (U.). Ausländerpolitik und Integration. Eine empirische Untersuchung der Rechtsprobleme von türkischen Arbeitnehmern und ihrer Familienangehörigen. (Politique à l'égard des étrangers et intégration. Etude empirique des problèmes juridiques auxquels sont confrontés les travailleurs turcs et les membres de leurs familles). Francfort/Berlin, 1982.

SCHULTZ (H.). Soziale Beziehungen im Alter. Integration durch Isolation, (Rapports sociaux de la vieilesse. L'intégration par l'isolement). Francfort/New York, 1979.

SCHULTE (A.) et coauteurs (édit.). Ausländer in der Bundesrepublick. Integration, Marginalisierung, Identität, (Les étrangers en République Fédérale. Intégration, marginalisation, identité). Francfort, 1985.

SCHRADER (A.), NIKLES (B.W.) et GRIESE (H.M.). Die Zweite Generation. Sozialisation und Akkulturation ausländischer Kinder in der Bundesrepublik. (La seconde génération. Socialisation et acculturation des enfants d’étrangers en République Fédérale). Königstein/Ts, 19792.

THOMAS (W.I.) et ZNANIECKI (F.). The Polish Peasant in Europe and America. Boston/New York, 2 vol., 1918-1921, 1958. 
TSIAKAI.OS (G.). Ausländerfeindlichkeit. Tatsachen und Erklärungsversuche, (La xénophobie. Réalités et tentatives d'explication). Munich, 1983.

TWENHOFEL (R.). Kulturkonflikt und Integration. Zur Kritik der Kulturkonfliktthese, (Conflits de cultures et intégration. A propos de la critique de la thèse des conflits de cultures), dans : HETTLAGE (R.) (édit.). Zuischenwelten der Gastarbeiter. Sondernummer der Schweizerischen Zeitschrift für Soziologie, (Les univers intermédiaires des travailleurs immigrés. Numéro spécial de la Revue suisse de sociologie), 10 (1984) 2. pp. $405-436$.

UNESCO (édit.). I.iving in Two Cultures. The Socio-Cultural Situation of Migrant Workers and Their Families. Paris (1982).

WEIDACHER (A.) et LOPEZ-BLASCO (A.). Ausländerpolitik und Integrationsforsforschung in der Bundesrepublik Deutschland. Eine Darstellung wichtigster Ergebnisse mit Auswahlbibliographie, (La politique à l’égard des étrangers et la recherche en matière d'intégration en République Fédérale d'Allemagne. Présentation des résultats les plus importants et choix bibliographique. Munich, 1982.

WEISCHE-ALEXA (P.). Sozial-kulturelle Probleme junger Türkinnen in der Bundesrepublik Deutschland. Mit einer Studie zum Freizeitverhalten türkischer Mädchen in Köln. (Difficultés socioculturelles des jeunes filles turques en République Fédérale d'Allemagne. Etude accompagnée d'une enquête sur le comportement des jeunes filles turques de Cologne dans leurs loisirs). Cologne, 1977

WILPERT (C.). International Migration and Ethnic Minorities : New Fields for Post-War Sociology in the Federal Republic of Germany, dans : Current Sociology 32 (1984) 3, pp. 305-352 (Migration in Europe, part 2, M. MOROKVASIC (édit.), 1984.

WURTINGER (H.) et BAATZ (R.). Wohnraumversorgung von Ausländern und Entballung überlasteter Gebiete durch städtebauliche MaBnahmen. Teil 1, (Lintervention de l'urbaniste dans la mise à la disposition des étrangers de locaux d'habitation et le décongestionnement des quartiers surpeuplés, première partie). Berlin, 1980.

WULFF (E.). Methodenfragen der vergleichenden Psychiatrie, (Questions de méthode en psychiatrie comparée) in du même auteur : Ethnopsychiatrie. Wiesbaden, 1978, pp. 70-77.

ZIERIS (E.). Wohnsituation ausländischer Arbeitnehmer und MaBnahmen zu ihrer Verbesserung im Urteil der Gemeinden. (Les conditions de logement des travailleurs étrangers et les mesures destinées à leur amélioration vues par les communes), dans Konrad-Adenauer-Stiftung. Fondation Konrad Adenauer (édit.). Studien zur Kommunalpolitik. Etudes de politique communale, vol. 16 (1977), pp. 185-282.

ZIMMERMANN (E.). Emigrationsland Süditalien. Eine kulturanthropologische und sozialpsychologische Analyse, (L'Italie méridionale, pays d'émigration. Etude d'anthropologie culturelle et de psychologie sociale). Tübingen, 1982. 